\title{
Tuberculosis of Tongue (Oral Cavity): A Case Report
}

\author{
Rajendran D Kumar ${ }^{1}$, Vithal D Udagatti ${ }^{2}$
}

\begin{abstract}
Oral lesions of tuberculosis, though uncommon, are often seen in both the primary and secondary stages of the disease. In secondary tuberculosis, the oral manifestations may be accompanied by lesions in the lungs, lymph nodes, or in any other part of the body and can be detected by a systemic examination. Primary oral tuberculosis may present as a diagnostic challenge for the clinician. Here we report a case of tuberculosis of oral cavity with primary in lung (a cavity lesion) and sputum positive for acid fast bacilli. However, the biopsy from the lesion was negative.
\end{abstract}

Keywords: Oral cavity, Tongue, Tuberculosis.

International Journal of Head and Neck Surgery (2018): 10.5005/jp-journals-10001-1356

\section{INTRODUCTION}

Tuberculosis is a chronic granulomatous disease that can affect various systems of the body. In humans, the disease is caused by Mycobacterium tuberculosis, Mycobacterium bovis species and a typical mycobacteria. ${ }^{1}$

Primary infection can also affect the pharynx, cervical lymph nodes, intestine, or oral mucosa. Secondary tuberculosis, which occurs from a healed primary focus or due to endogenous spread of the infection, is usually chronic in nature and can cause considerable destruction of the involved tissue with caseation, cavity formation, and fibrosis. Both primary and secondary types of tuberculosis can cause lesions in the oral cavity. In secondary tuberculosis, lesions of the oral cavity may accompany lesions in the pharynx, lungs, lymph nodes, or skin. ${ }^{1}$

Pulmonary tuberculosis is the most common form of the disease. However, it can also occur in the lymph nodes, meninges, kidneys, bone, skin, and oral cavity. The disease spreads by inhalation of the bacilli through dust or droplets. Initial infection often causes small parenchymatous lesions in the lungs, draining lymphatics and the lymph nodes. These three components together form the primary complex of tuberculosis. ${ }^{2}$

Here we report a case of secondary tuberculosis of tongue with primary foci in lungs.

\section{Case Description}

A 50-year-old man presented to the Department of ENT complaining of an ulcer in the mouth which had been present for the past four months and was gradually increasing in size (Fig. 1). He did not have any systemic complaints, was not on any medications, and had no history of any allergy. He was a chronic smoker and was unable to give up the habit. Physical examination showed a single discrete ulcer of less than $1 \mathrm{~cm}$ in diameter on the right lateral side of the tongue with irregular margins, spreading to the tip and dorsal surface and floor of the mouth. The ulcer was bordered by welldefined margins around which there were several small nodular swellings. On palpation, the base of the ulcer was tender with indurated margins, firm but not fixed to the underlining muscle and covered with slough.

A provisional diagnosis of malignancy was kept in mind. General examination revealed no lymphadenopathy, but on auscultation
${ }^{1}$ Department of ENT and HNS, RajaRajeswari Medical College and Hospital, Bengaluru, Karnataka, India

${ }^{2}$ Department of ENT, NRM ENT Hospital, Raichur, Karnataka, India

Corresponding Author: Rajendran D Kumar, Department of ENT and HNS, RajaRajeswari Medical College and Hospital, Bengaluru, Karnataka, India, Phone: +91 9620928650, e-mail: dinuraj1186@gmail.com

How to cite this article: Kumar RD, Udagatti VD. Tuberculosis of Tongue (Oral Cavity): A Case Report. Int J Head Neck Surg 2018;9(4):121-123.

Source of support: Nil

Conflict of interest: None

of chest, crepitation was present in bilateral lung field. Blood investigation showed a raised erythrocyte sedimentation rate level, ELISA for HIV nonreactive and VDRL negative. Chest X-ray showed extensive bilateral lesions with some areas of cavitation suggestive of tuberculosis (Fig. 2). Sputum samples for acid fast bacilli were positive.

A deep punch biopsy of the tongue revealed multiple confluent and discrete granulomas composed of epithelioid histiocytes and Langhans giant cells with central caseous necrosis (Fig. 3). The diagnosis of tuberculosis of the tongue secondary to primary sputum positive lung tuberculosis was made. The patient was on anti-tuberculosis drugs and was advised to stop smoking. Later, on follow-up after 6 months, the oral ulcer had healed.

\section{Discussion}

The tongue is frequently exposed to a heavy concentration of tubercle bacilli from the sputum of the patient with pulmonary tuberculosis. The bacilli do not involve the intact mucosa of the tongue or oral cavity. Farber et al. indicated that less than $0.1 \%$ of tuberculous patients exhibited oral lesions. ${ }^{3}$

In primary oral tuberculosis, the organisms are directly inoculated on the oral mucous membrane of a person who has not been infected. In the secondary type, oral tuberculosis usually coexists with pulmonary disease. Self-inoculation may take place due to the infected sputum or hematogenous seeding. However, the role of trauma cannot be underestimated as the stratified squamous epithelium of the oral cavity normally resists direct penetration by tubercle bacilli. The local resistance may be due 

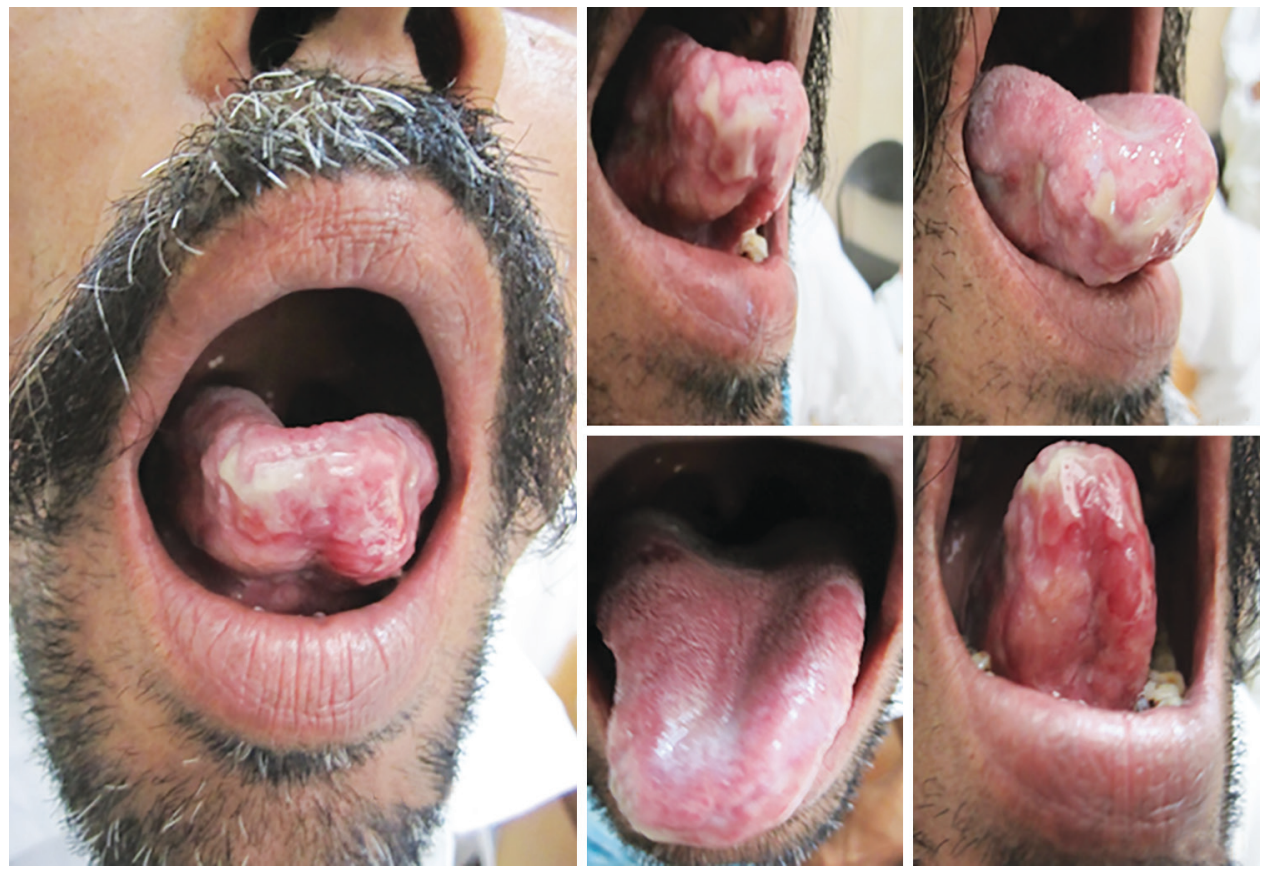

Fig. 1: Tongue showing the ulcer
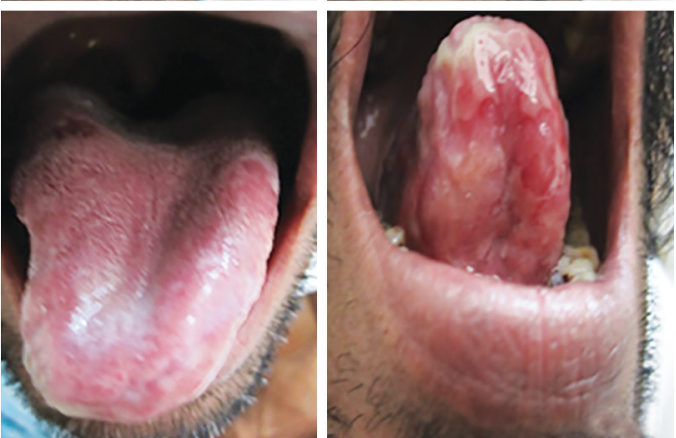

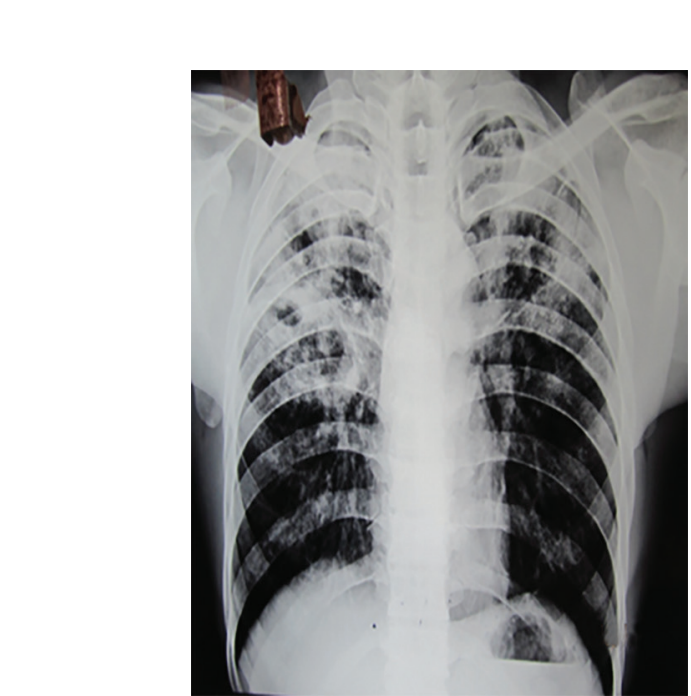

Fig. 2: Chest X-ray: Cavity lesions in both lung fields at the upper and mid-zone

to stratified squamous epithelium, regular cleaning by saliva, and paucity of lymphoid tissue in the anterior part of the tongue. ${ }^{4}$

Secondary tuberculosis of the tongue manifests in two ways: one with shallow, oval, indolent, painful ulcers with overhung margins and the other with a circumscribed interstitial tuberculoma. Both types of lesions occur in advanced untreated cases of tuberculosis. ${ }^{4}$

Three forms of oral tuberculosis have been described, namely, acute miliary, chronic ulcerative, and lupus vulgaris. About $93 \%$ of oral lesions are ulcerative and approximately half of which affect tongue. A diagnosis of tuberculosis is made by biopsy; the deeper tissue must be included as the granulomas are seen in deeper dermis. All chronic ulcerative lesions should be thought of as tuberculosis lesions along with malignancy which is much more common. ${ }^{5}$

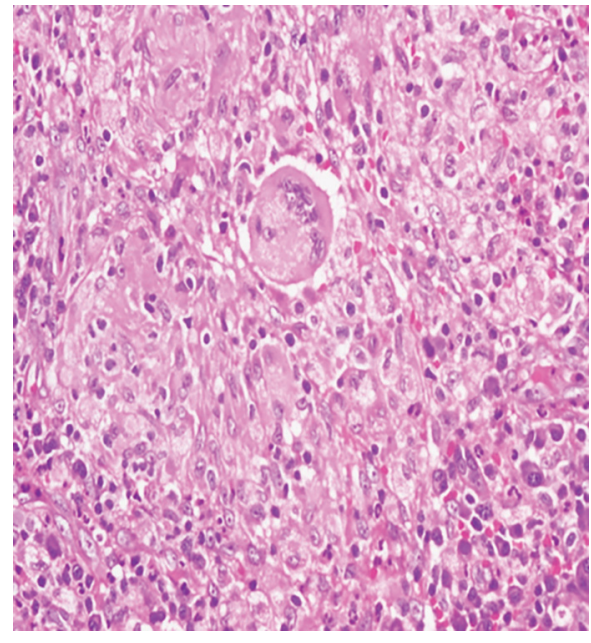

Fig. 3: Histopathological examination of the biopsy, suggestive of multiple confluent and discrete granulomas composed of epithelioid histiocytes and Langhans giant cells with central caseous necrosis

\section{Conclusion}

Tuberculous ulcers of the tongue occur on the tip, lateral borders, dorsum, towards the midline and base. They are irregular, with undermined margins, pale, indolent, with granulations in the floor and sometimes with a thin slough. Differential diagnosis of oral ulcers includes aphthous ulcer, traumatic ulcer, actinomycosis, syphilitic ulcer, Wegener's granuloma, and carcinoma. Diagnosis is made by identification of caseating granuloma on biopsy. Deeper biopsies are always advocated for ulcers of the tongue since superficial biopsies may not reveal the etiology due to epithelial hyperplasia. Most often, complete remission of tuberculous ulceration of the tongue takes place along with a remission of lung lesions after adequate chemotherapy. ${ }^{4-7}$ 


\section{References}

1. Prabbu SR, Wilson DF, et al. Oral Diseases in the Tropics. Oxford University Press; 1993.

2. Topazian RG, Goldberg MH. Oral and Maxillofacial Infections, 2nd ed., WB Saunders Co.; 1987. p. 413.

3. Fraber JE, Friedland E, et al. Tuberculosis of the tongue. Am Rev Tuber 1940;42:766.

4. Fujiboyashi T, Takashi $Y$, et al. Tuberculosis of the tongue. A case report with immunologic study. Oral Surg Oral Med
Oral Pathol 1979;47(5):427-435. DOI: 10.1016/0030-4220(79) 90124-5.

5. Bhatt AP, Dholakia HM. Tuberculosis of oral Mucosa. J Indian Dent Assoc 1974;46:161.

6. Laws IM. Oral tuberculosis. Case reports. Br Dent J 1973;134:146-148. DOI: 10.1038/sj.bdj.4802971.

7. De Lathouwer C, Cauchre $C$, et al. A rare and complex case of multifocal mucocutaneous lupus tuberculosis with isolated lesion of the tongue. Oral Surg Oral Med Oral Pathol 1975;39(2):211-215. DOI: $10.1016 / 0030-4220(75) 90222-4$. 